\title{
Morphological effect on the electrochemical behavior of electric double-layer capacitors
}

\author{
M. Endo, ${ }^{\text {a) }}$ Y.J. Kim, T. Maeda, K. Koshiba, and K. Katayam \\ Faculty of Engineering, Shinshu University, 4-17-1 Wakasato, Nagano 380-8533, Japan \\ M.S. Dresselhaus \\ Department of Physics and Department of Electrical Engineering, and Computer Science, \\ Massachusetts Institute of Technology, Cambridge, Massachusetts 02139
}

(Received 14 May 2001; accepted 6 September 2001)

\begin{abstract}
Activated milled mesophase carbon fibers (AC-mMPCF, MP-series) show a higher specific capacitance in spite of a smaller specific surface area than those of powder-type activated carbons (AC-series). This phenomenon can be interpreted to mean that it is difficult to predict the capacitance of an electric double-layer capacitor (EDLC) knowing just the surface area and the pore size. More information is needed about other inherent characteristics of the samples, for example, the equivalent series resistance (ESR), shape of the pores, etc. We investigate here other characteristics of the samples. Consequently, it was deduced that the MP-series of EDLCs have a slit pore shape, which affects the accessibility of the electrolyte ion onto the electrode surface. Moreover, the MP-series of materials have suitable ESR values, and these material properties themselves should be considered as factors that affects the deterioration of the specific capacitance of EDLCs.
\end{abstract}

\section{INTRODUCTION}

The electrochemical double layer capacitor (EDLC) is a new type of capacitor, offering new features, with characteristics between those of a battery and a simple capacitor. $^{1,2}$ In general, positive and negative electrical charges are arrayed in opposite positions with an extremely short distance between two different phases (e.g., the solid electrode and the liquid). This charge distribution layer is called the electric double layer. There are various explanations for this interfacial charge distribution. ${ }^{3-9}$

EDLCs typically consist of a pair of polarizable electrodes, a separator, and an aqueous or organic electrolyte. ${ }^{10,11}$ Although there are plenty of parameters to optimize and variations to be tried, the most important source of variation of the capacitance may be the electrode material itself. The capacitive storage comes from the electrochemical double layer formed at the electrode/ electrolyte interface. Therefore, the specific capacitance of EDLC electrodes are linked to the physical area, the pore size, the ionic accessibility of the electrode material, and the electrolyte properties. Recently, activated carbon (AC) and activated carbon fiber (ACF) research has

\footnotetext{
a) Address all correspondence to this author.
} e-mail: yjk@endomoribu.shinshu-u.ac.jp stimulated interest in these materials as electrodes of EDLC, because of their rich pore structures, which form within the electric double layers, and because of the relatively high conductivity of carbon.

The samples chosen as precursors in this study are coconut shell and milled mesophase pitch. Both materials have quite different morphologies and both are used generally. Coconut shell is a well-known precursor for the preparation of high-quality activated carbons. ${ }^{12}$ However, the other sample, activated milled mesophase carbon fiber (AC-mMPCF), was prepared by a melt-blown spinning process, with mesophase pitch before the activation process, instead of graphitization. Therefore, ACmMPCFs have a fiber shape. Furthermore, AC-mMPCFs are very interesting from a scientific viewpoint and are important for adsorbent studies from both a practical and fundamental perspective. ${ }^{13-15} \mathrm{AC}-\mathrm{mMPCF}$ have a more uniform pore size and a greater pore volume than usual granular AC material. The ACFs made from cellulose (CEL), polyacrylonitrile (PAN), and phenol-resin (PR) precursors have no mesopores and have only uniform micropores. The characteristics of these carbons are affected by the type of raw material and the manufacturing conditions. ${ }^{16}$

The aim of this paper is to investigate the performance characteristics of samples with different morphologies in electrochemical capacitor and to analyze the correlations between the physical and electrochemical properties, 
such as the specific surface area (SSA), the pore-size distribution (PSD), equivalent series resistance (ESR), pore shape, and so on.

\section{EXPERIMENTAL}

\section{A. Sample preparation}

The samples used in this study consist of a series of activated carbon (AC and MP) samples, each with a different surface area. These ACs were commercially prepared by Nippon Gaisi Co., Nagoya, Japan, by carbonizing and activating a coconut shell in steam/carbon dioxide. The AC-series used in this study were AC-a, AC-b, AC-c, and had Brunaver-Emmett-Teller (BET) specific surface areas of $959.7,1499.9$, and $1848.4 \mathrm{~m}^{2} / \mathrm{g}$, respectively. The surface area measurements were carried out using the standard BET method applied to an experimental adsorption over a range of relative pressures up to 1 . The adsorption isotherms were measured with a Gemini II 2375 instrument (Micromeritics, Tokyo, Japan) and nitrogen at $77 \mathrm{~K}$.

Other samples studied include milled fiber-type activated carbon fibers (AC-mMPCF), supplied by the Petoca Co., Japan, and prepared by carbonizing and activating a mesophase carbon fiber (MPCF). The mesophase pitch-based carbon fibers were obtained by a meltblown method (the average fiber diameter was $13 \mu \mathrm{m}$ ) from a mesophase pitch with a softening point of $290{ }^{\circ} \mathrm{C}$. After stabilization was performed under an air ambient atmosphere, the carbonization was performed at 500$800{ }^{\circ} \mathrm{C}$. Moreover, these carbon fibers were milled mechanically and their mean length was selected to be about $25 \mu \mathrm{m}$. The fibers were well mixed with $200 \mathrm{wt} \%$ potassium hydroxide $(\mathrm{KOH})$, which was used as an activator. The temperature ramp-up for the activation was carried out with a fixed programming rate in the nitrogen air current, and a predetermined retention time was used at each temperature in the ramp-up. After activation the sample was cooled in a nitrogen air current. The samples thus obtained were washed with pure distilled water to eliminate the acid remains, and finally the samples were dried at an appropriated temperature. Sample inner diameter was MP-a and MP-b, and it had a surface area of 770.7 and $683.3 \mathrm{~m}^{2} / \mathrm{g}$, respectively.

TABLE I. Specification of coin cell AC-series and MP-series.

\begin{tabular}{ccccc}
\hline $\begin{array}{l}\text { Sample } \\
\text { ID }\end{array}$ & $\begin{array}{c}\text { Weight } \\
(\mathrm{mg})\end{array}$ & $\begin{array}{c}\text { Volume } \\
(\mathrm{m} \mathrm{cc})\end{array}$ & $\begin{array}{c}\text { Density } \\
(\mathrm{g} / \mathrm{cc})\end{array}$ & $\begin{array}{c}\text { Diameter } \\
(\mathrm{mm})\end{array}$ \\
\hline AC-a & 80 & 120 & 0.66 & 13.45 \\
AC-b & 77 & 140 & 0.55 & 13.45 \\
AC-c & 86 & 160 & 0.54 & 13.45 \\
MP-a & 80 & 110 & 0.73 & 13.45 \\
MP-b & 82 & 110 & 0.75 & 13.45 \\
\hline \hline
\end{tabular}

\section{B. Cell configuration and capacitance measurement}

To determine the properties of the electric double layer formed on the electrode, a configuration of electrode/ electrolytic solution/electrode was selected. The test cell was composed of a pair of electrodes, prepared from ACs

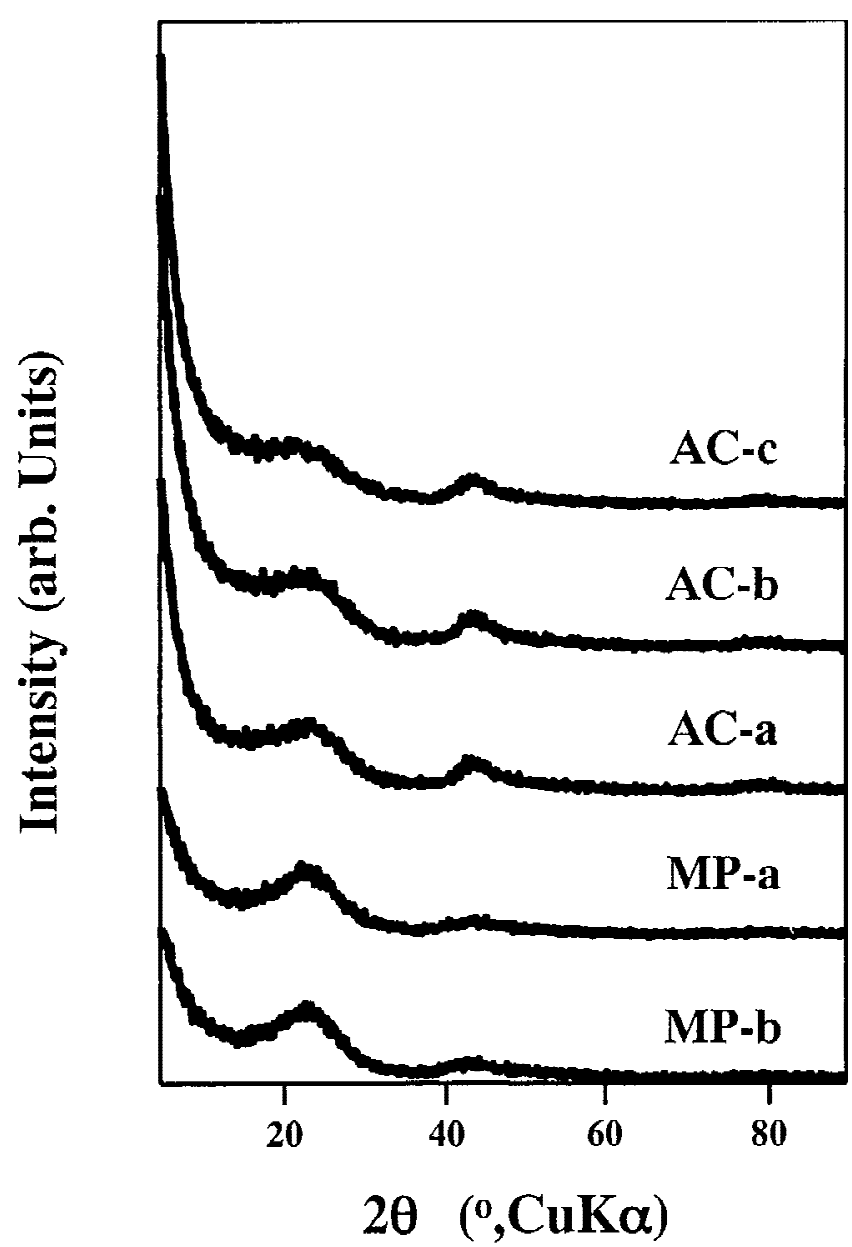

FIG. 1. X-ray diffraction profiles of activated carbons (AC-series) and activated carbon fibers (MP-series).

TABLE II. Crystallographic properties of MP-series and AC-series samples obtained by XRD.

\begin{tabular}{ccccccc}
\hline \hline Sample ID & $\mathrm{d}_{(002)}{ }^{\mathrm{a}}$ & $\mathrm{L}_{\mathrm{c}(002)}{ }^{\mathrm{b}}$ & $\mathrm{L}_{\mathrm{a}(10)}{ }^{\mathrm{c}}$ & $\mathrm{FWHM}_{(002)}{ }^{\mathrm{d}}$ & $2 \theta_{(002)}$ & $2 \theta_{(10)}$ \\
\hline MP-a & 3.86 & 11.23 & 48.94 & 7.9 & 23.02 & 43.58 \\
MP-b & 3.85 & 12.09 & 48.94 & 7.3 & 23.09 & 43.59 \\
AC-a & 3.84 & 10.48 & 69.97 & 8.3 & 23.16 & 43.79 \\
AC-b & 3.82 & 11.23 & 65.30 & 7.8 & 23.29 & 43.79 \\
AC-c & 3.82 & 11.23 & 69.97 & 7.8 & 23.29 & 43.79 \\
\hline \hline
\end{tabular}

${ }^{\mathrm{a}} \mathrm{d}=\mathrm{n} \lambda / 2 \sin \theta, \mathrm{v}=1, \lambda=1.5405 \AA$.

${ }^{\mathrm{b}} \mathrm{L}_{\mathrm{c}(002)}=\mathrm{n} \lambda / \beta \cos \theta, n=1$.

${ }^{c} L_{\mathrm{a}(10)}=\mathrm{n} \lambda / \beta_{1 / 2} \cos \theta, n=1.77$.

${ }^{\mathrm{d}}$ Full-width of half maximum. 
and ACFs, and glass-paper was sandwiched as a separator between the pair of electrodes so that electrodes were facing each other. Samples used ACs and ACFs mixed with $5 \mathrm{wt} \%$ poly-tetrafluoroethylene (PTFE) as a binder. Then coin-type electrodes were prepared by pressurization to $1 \mathrm{ton} / \mathrm{cm}^{2}$. The prepared electrodes had a dimension of $13.4 \mathrm{~mm}$ in diameter and around $0.3 \mathrm{~mm}$ in thickness. The dimensions of the prepared samples are summarized in Table I. A mixture of propylene carbonate (PC) with tetraethylammonium tetrafluoroborate $\left(\mathrm{Et}_{4} \mathrm{NBF}_{4}\right)$ was used as an electrolytic solution.

The capacitor was charged at a constant voltage (2.5, 3.0 , and $3.5 \mathrm{~V}$ ) for $2 \mathrm{~min}$ and then discharged at a constant current from 1 to $40 \mathrm{~mA}$. Its capacitance $\mathrm{C}$ was calculated from the equation $C=(I \times t) / V$, where $I$ is the discharge current, $t$ is the time for discharge for the voltage change from $40 \%$ to $60 \%$ of final charge voltage, and $V$ is final charge voltage, that is, $2.5,3.0$, and $3.5 \mathrm{~V}$.

\section{Sample characteristics}

The high-resolution scanning electron microscope (SEM) measurements were performed with an acceleration voltage as low as $5 \mathrm{kV}$ (Hitachi S-4200, Tokyo, Japan) to obtain sufficient resolution to study the morphology of the fibers. An x-ray diffractometer (Rigaku RINT 2100, 40kV, $30 \mathrm{~mA}$, Tokyo, Japan) with a $\mathrm{CuK}_{\alpha}$ $(\lambda=0.15406 \mathrm{~nm})$ source was used to measure the Bragg reflections from ACs and ACFs.
The lattice parameters $L_{c}$ and $L_{a}$ of the samples were determined from the widths of the (002) and (001) diffraction lines, respectively.

Image analysis of the transmission electron microscopy (TEM) photographs was used extensively here as a powerful method to analyze the PSD. ${ }^{17,18}$ Twodimensional fast Fourier transforms (FFTs) were carried out on the TEM (JEM-2010, JEOL, Tokyo, Japan) images $(512 \times 512$ pixels $)$ with our image processing system, and the PSD was obtained by calculation of the power spectrum with a Silicon (Nippon SGI, Tokyo, Japan) graphics system.

\section{RESULTS AND DISCUSSION}

Figure 1 shows the $\mathrm{x}$-ray diffraction (XRD) profiles of both the AC-series and the MP-series. All samples show a broad $\mathrm{d}_{\mathrm{O02}}$ line, indicating a typical nongraphitizable carbon. There are two broad lines in the XRD patterns near $2 \theta \cong 23^{\circ}$ and $44^{\circ}$, corresponding to the (002) and (10) peaks, respectively. The computed magnitude of $\mathrm{L}_{\mathrm{c}}$ (stack height) and $\mathrm{d}_{002}(d$-spacing) are $10-11 \AA$ in the AC-series samples and 11-12 $\AA$ in the MP-series samples, respectively. These materials have no 3-dimensional structure because the (10) peak shows no splitting into (100) and (101) structures, and moreover the (112) peak around $82^{\circ}$ is hardly observed. Consequently, XRD studies confirmed that the crystallographic properties of both samples have nearly same structure, and we can hardly

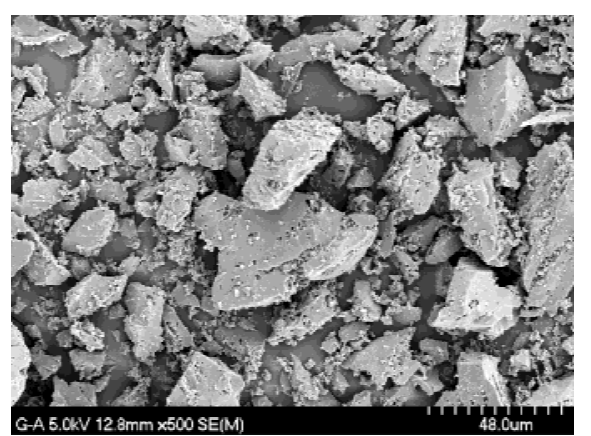

(a)

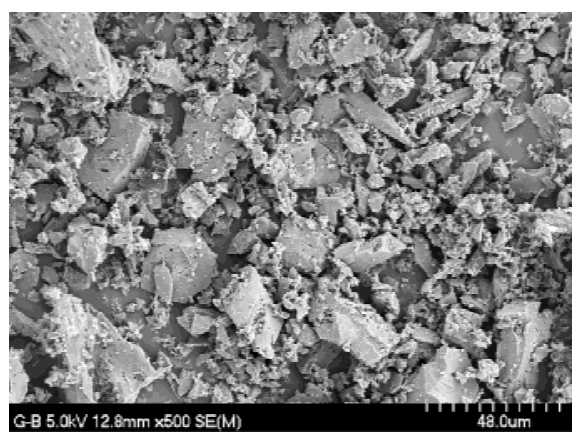

(b)

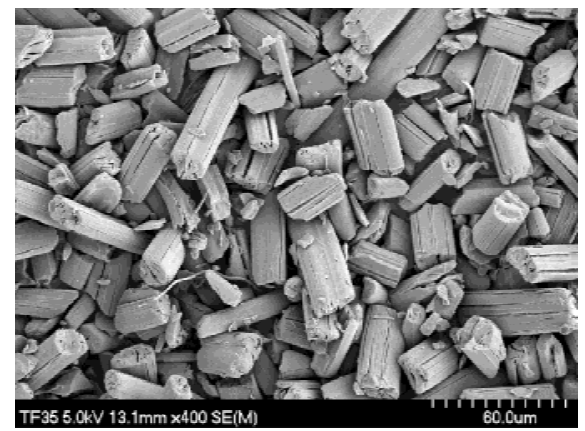

(d)

(e)

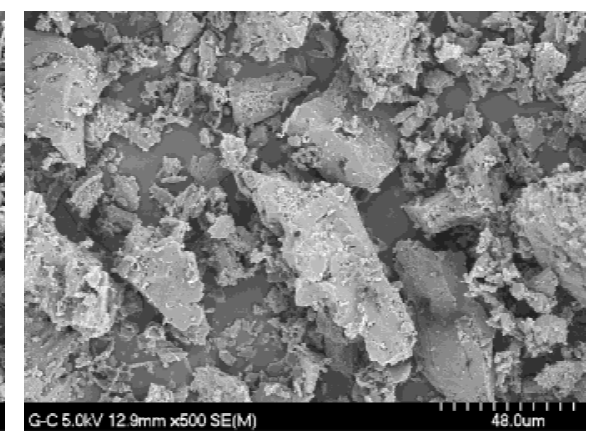

(c)

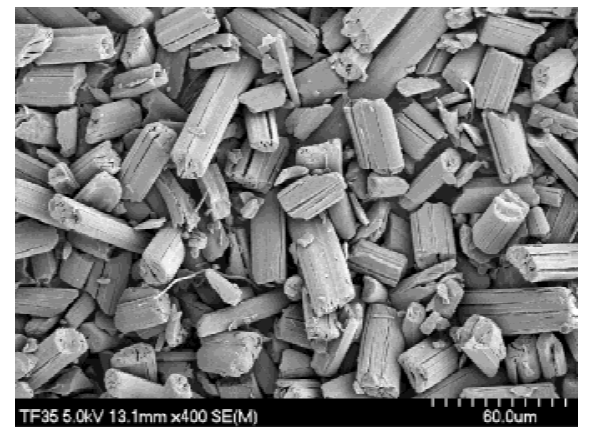

FIG. 2. Low-magnification SEM photographs of the (a, b, c) AC-series and (d, e) MP-series. 
distinguish any difference between them. The detailed data that we could obtain from the XRD measurements is summarized in Table II.

Figure 2 shows a low-magnification SEM image of powder-type activated carbons and activated milled mesophase carbon fibers, the images were made at $\times 500$ and $\times 400$, respectively. Three samples of the AC-series have a random distribution of powder sizes, and besides, it is observed that there are also a lot of extraneous materials. The AC-mMPCFs are randomly arranged, and both samples include a smaller amount of dustlike broken carbon, resulting from the milling process. It is noteworthy that the fibers can be cut rather homogeneously with a length of about several tens of millimeters and that split fibers are observed very seldom. Figure 2 confirms that the two kinds of samples have quite a different morphology.
To investigate the surface morphology further, SEM photographs were taken at a higher magnification. Figure 3 shows high-resolution (HR) SEM photographs of ACseries carbons made from coconut shell at a magnification of $\times 50,000$ [Figs. 3(a)-3(c)] and $\times 100,000$ [Figs. 3(d)-3(f)] respectively, and Figs. 3(a) and 3(d) are the photographs of AC-a, while Figs. 3(b) and 3(e) are photographs of AC-b, and Figs. 3(c) and 3(f) are photographs of AC-c. Generally, powder-type activated carbons, such as the AC-series, show a tendency that the pore size, as well as the number of pores, increases as the surface area increases. Almost all the pores could be observed in $\mathrm{AC}-\mathrm{a}$, and these pores show a size under the meso-size (below $500 \AA$ ), but a lot of macropores could be observed as the temperature of the activation process increases.

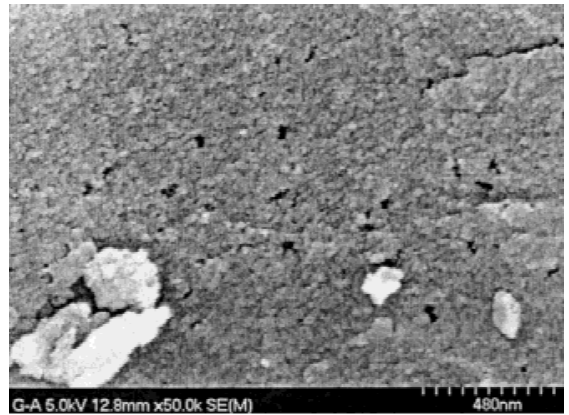

(a)

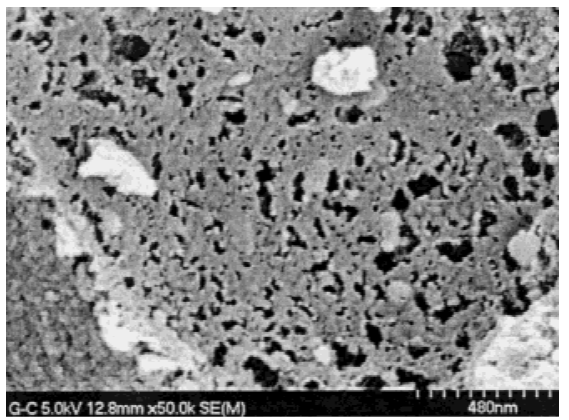

(c)

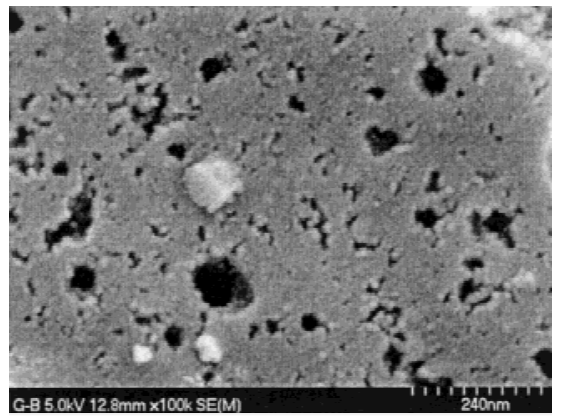

(e)

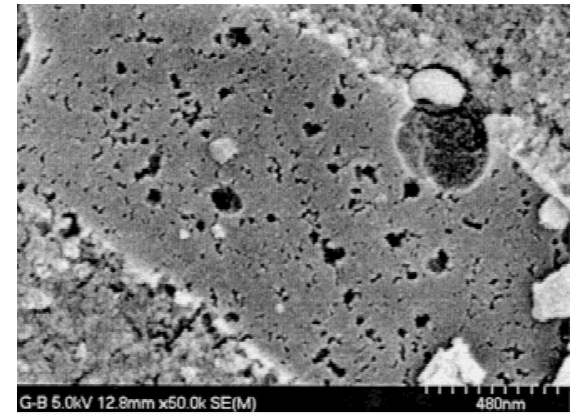

(b)

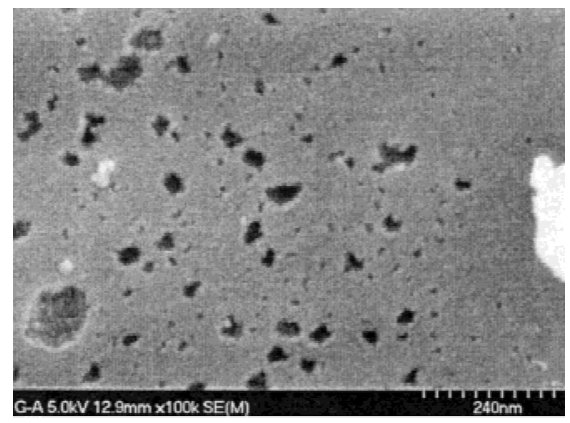

(d)

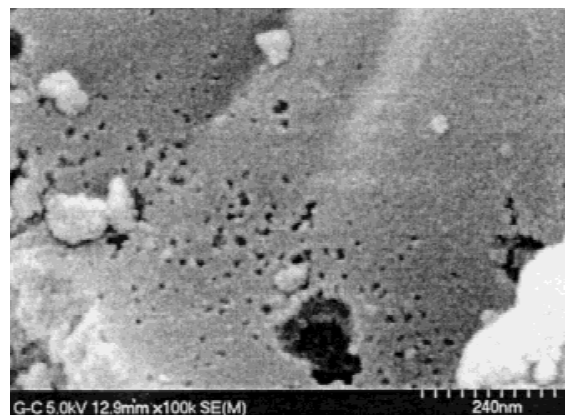

(f)

FIG. 3. HR-SEM photographs of AC-series made from coconut shell: (a, d) is the photographs of AC-a, (b, e) is AC-b, (c, f) is AC-c. Each picture was taken at a magnification of $\times 50,000$ and $\times 100,000$ for each sample. 
Figure 4 shows HR-SEM photographs of the center and periphery of an AC-mMPCF. Figs 4(a) and 4(d) show the overall features of the MP-series, Figs. 4(b) and 4(e) illustrate the periphery, and Figs. 4(c) and 4(f), illustrate the center of the fiber. The structure of nonactivated pristine MPCF has already been observed and demonstrated by Endo et al. ${ }^{19}$ MPCFs have quite a different morphology between the center and the periphery. That is, the cross-sectional core region has a well-aligned layer structure, while the periphery of the core is distinguished as having a more wavy layered structure. Moreover, its original morphology remains preserved after the activation process. The postulated form of the porous structure of AC-mMPCFs is supported by its lamellae arrangement, ${ }^{20}$ where individual lamellae or their groupings are interbonded by cohesive forces. Due to the unevenness of the surfaces of the lamellae, free spaces of molecular dimensions remain between the contact points, which can be defined as slit-shaped micropores. The micropores have outlets on the surfaces of the meso- and macropores, and therefore, the number of accessible micropores, proportional to surface $S_{\text {micro }}$, depends on the surface area of the meso- and macropores included in the value of $S_{\mathrm{BET}}{ }^{21}$

Table III summarizes the characteristic properties obtained by the BET analyses. As mentioned above, in both series of samples, the micropore volume increases as the specific surface area increases. AC-c shows specific surface area as high as $1848 \mathrm{~m}^{2} / \mathrm{g}$. The MP-series shows a specific surface area of $683,770 \mathrm{~m}^{2} / \mathrm{g}$ lower than that of the AC-c series. Figure 5 shows adsorption isotherms. The nitrogen adsorption isotherms are of

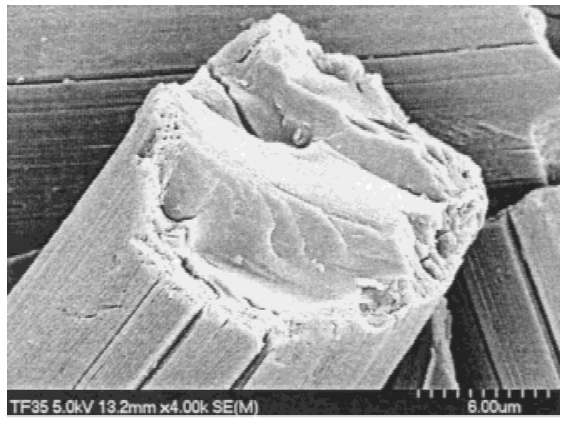

(a)

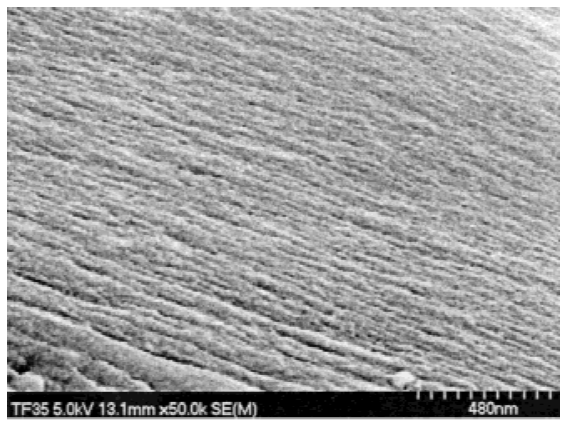

(c)

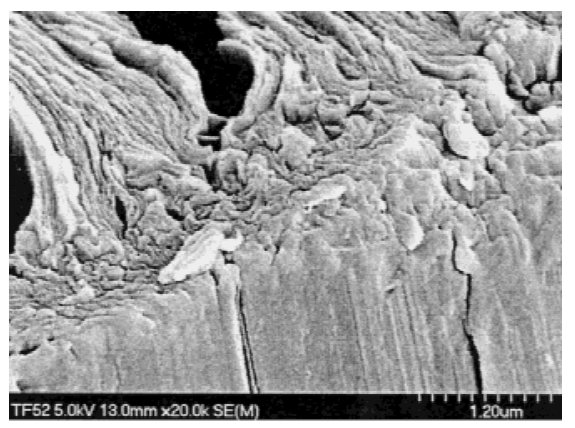

(e)

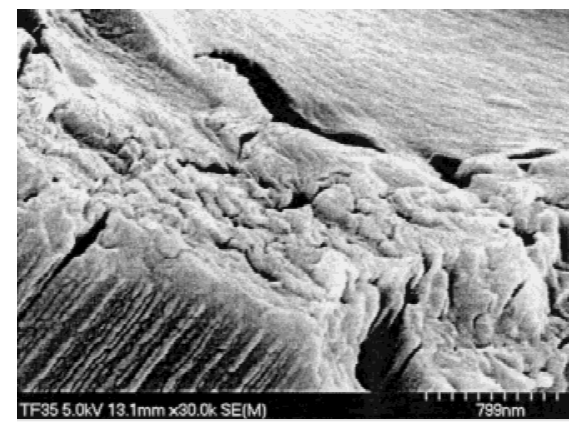

(b)

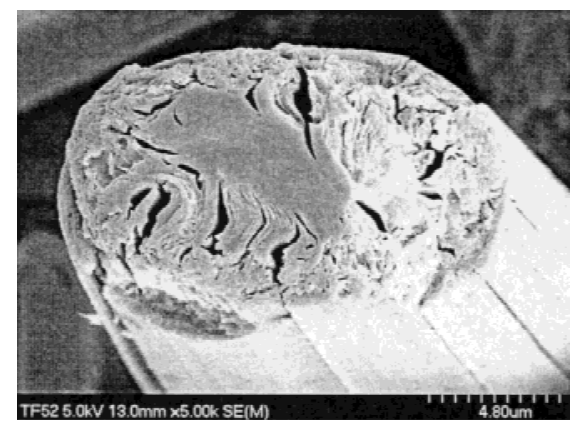

(d)

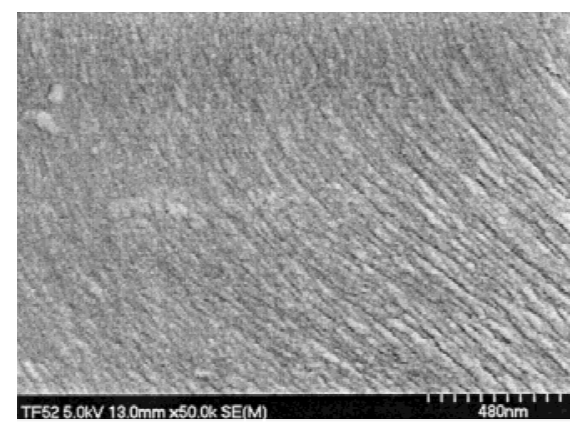

(f)

FIG. 4. HR-SEM photographs of the MP-series made from mesophase carbon fiber. (a, b, c) are photographs of MP-a, and taken at the magnification of $\times 4,000, \times 30,000$, and $\times 50,000$ in order. $(d, e, f)$ are the photographs of MP-b, and taken at the magnification of $\times 5,000, \times 20,000$, and $\times 50,000$, respectively. 
TABLE III. BET properties of the AC-series and MP-series samples.

\begin{tabular}{cccc}
\hline \hline Sample ID & $\begin{array}{c}\text { Surface area } \\
\left(\mathrm{cm}^{2} / \mathrm{g}\right)\end{array}$ & $\begin{array}{c}\text { Total pore } \\
\text { volume } \\
\left(\mathrm{cm}^{3} / \mathrm{g}\right)\end{array}$ & $\begin{array}{c}\text { Average pore } \\
\text { diameter } \\
(\AA)\end{array}$ \\
\hline AC-a & 959.73 & 0.52 & 14.67 \\
AC-b & 1499.91 & 0.82 & 14.70 \\
AC-c & 1848.37 & 0.98 & 14.15 \\
MP-a & 770.73 & 0.42 & 14.68 \\
MP-b & 683.33 & 0.37 & 14.52 \\
\hline \hline
\end{tabular}

the BET I type and have no hysteresis, indicating the presence of micropores only. The micropore size distribution obtained by the MP-method ${ }^{22}$ is compared with the PSD profile obtained by image analysis of TEM photographs.

Figure 6 shows the pore size distribution evaluated by both methods of conventional $\mathrm{N}_{2}$ adsorption and image analysis of TEM photographs. The TEM photographs were taken at a magnification of $\times 200,000$ in all samples. The TEM image exhibited a random structure, unlike that seen in crystalline graphite, and there is no particular orientation in the pore arrangement shown in the image even in the samples in the fiber shape. From this result, it is confirmed that the pore size increases with the increase of surface area in both methods. But the two profiles evaluated under the micropore size (under $20 \AA$ ) do not agree well. The PSDs obtained by the image analysis are as follows: the AC-series [Figs. 6(a)-6(c)] have a maximum peak at $18 \AA$, at 24 and $17 \AA$, and at $20-38 \AA$. The MP-series [Figs. 6(d) and 6(e)] have a maximum peak around 20 and $17 \AA$, so that the MP-a series has a littler larger pore size than the MP-b series.

Figure 7 shows the charge-discharge profiles (discharged at $1 \mathrm{~mA} / \mathrm{cm}^{2}$ of current density), when the electrode of electronic double layer capacitor is charged at $3.5 \mathrm{~V}$ and then discharged at $1 \mathrm{~mA} / \mathrm{cm}^{2}$ of current density. The discharge curves consist of an initial steep voltage drop followed by a slow and almost linear voltage decrease with time. In the AC-series, the discharging time decreased with increasing specific surface area. However, even though the MP-series samples have a much smaller specific surface area, these data show a longer discharging time. As for MP-a, with $770 \mathrm{~m}^{2} / \mathrm{g}$ of surface area, the discharge time from the initial voltage drop to $1.49 \mathrm{~V}$ took around $87 \mathrm{~min}$. AC-c, having $1848 \mathrm{~m}^{2} / \mathrm{g}$ surface area, took somewhat less time, around $75 \mathrm{~min}$, for discharge. This comparison shows the possibility of application as a cappatery (the concept for a hybrid between a capacitor and a battery), which has the properties of a capacitor and a battery by the control of discharge current density (discharge time could be controlled by the current density when it was discharged).

Figure 8 shows the specific capacitance per (a) weight and (b) volume obtained at a current density of 1 and $10 \mathrm{~mA} / \mathrm{cm}^{2}$ as the charge voltage increases. The specific capacitance per weight and volume both increase when the charging voltage is increased for both discharge current densities. MP-a has as high as $40.9 \mathrm{~F} / \mathrm{g}$ capacitance when it is charged until $3.5 \mathrm{~V}$ and discharged at $1 \mathrm{~mA} / \mathrm{cm}^{2}$. Moreover, interestingly, when the discharge is performed at $10 \mathrm{~mA} / \mathrm{cm}^{2}$, which means a larger amount of electron current, $46 \mathrm{~F} / \mathrm{g}$ of capacitance could be obtained. However, for the AC-series, despite the higher specific surface area, $32 \mathrm{~F} / \mathrm{g}$ of capacitance is obtained at both the discharge current densities for the AC-c case. The others (AC-b, AC-a) show increased capacitance with increasing charge voltage, the same as the MP-series did. Such a phenomena may be related to the accessibility of the electrolyte ion. It is well known that the ACFs have a porous structure, having only micropores, with exposure directly to the exterior of the material. ${ }^{16}$ With that structure it is easy to insert or remove the electrolyte ion. Besides, with mesophase pitch it is easy to construct a ribbonlike planar layer, so it is inferred that the pore structures from the MP-series of samples have a slit shape. ${ }^{21}$ The pore shape can help to prevent the bottleneck effect (The present bottle-neck condition was compared to the traffic congestion, which happens if the electrolyte ions gather simultaneously at a narrow entrance like the neck of a bottle) of the electrolyte ion, when the cell is charged or discharged.

Figure 9 shows the variation of the ESR with discharge current density variation when the cell is charged until 3.5 V. As shown in the figure, AC-a has the largest ESR value, that is, the worse conductivity. Both MPseries samples show ESR values as high as about $28 \Omega$, independent of current density.

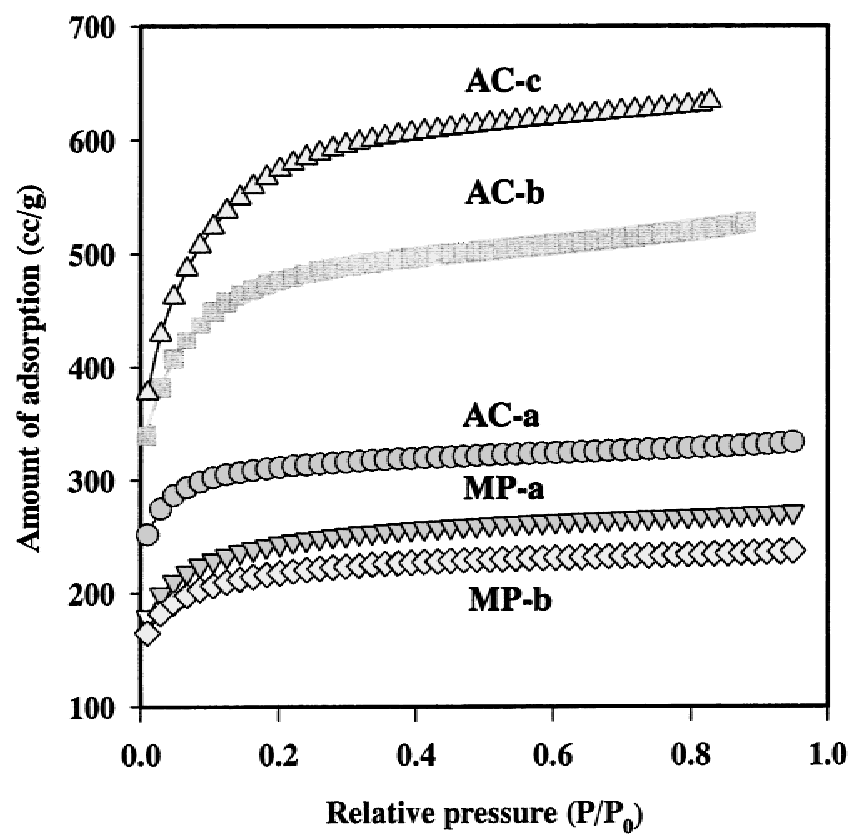

FIG. 5. Adsorption isotherms of AC-series and MP-series obtained by the BET method. 


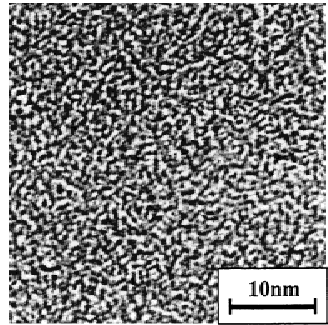

(a)

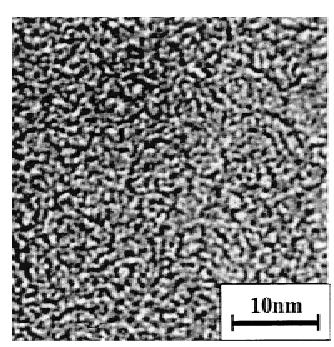

(b)

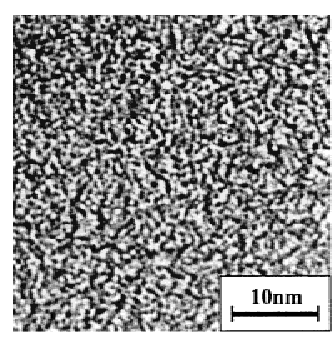

(c)

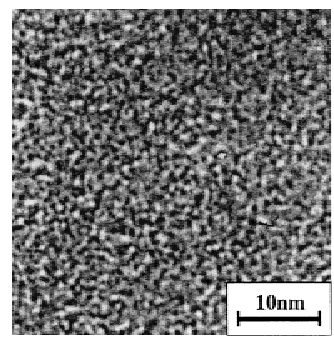

(d)

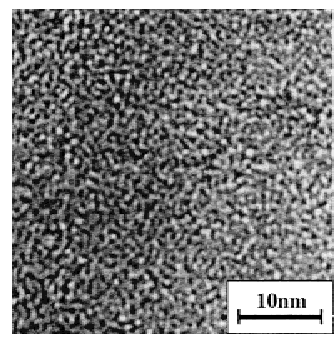

(e)

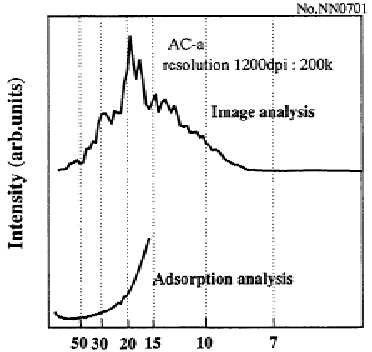

Pore diameter ( $(\AA)$

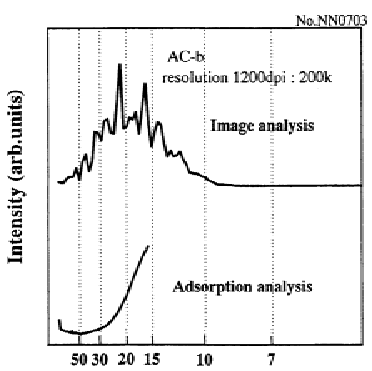

Pore diameter ( $(\AA)$

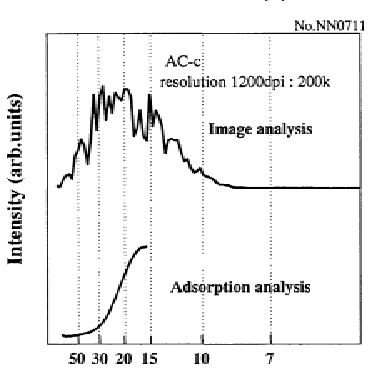

Pore diameter $(\tilde{\mathbf{A}})$

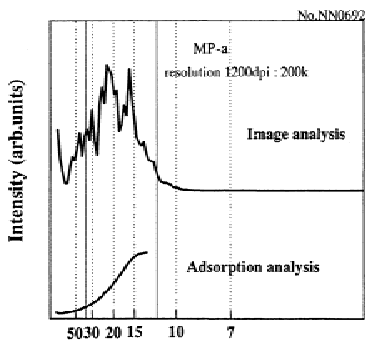

Pore diameter $(\AA)$

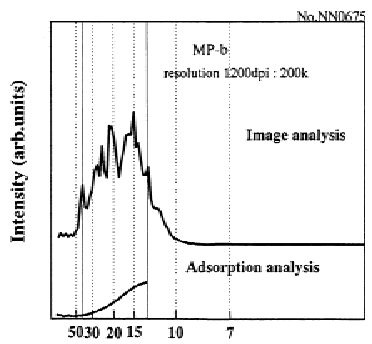

Pore diameter $(\dot{A})$
FIG. 6. Comparison of the pore size distributions obtained by the BET method and image analysis. (a, b, c) are the results for the AC-series, and $(\mathrm{d}, \mathrm{e})$ are the results for the MP-series.

Figure 10 illustrates the accessibility of the MP-series and $\mathrm{AC}$-series samples, which is calculated by dividing the specific capacitance (SC) by the BET surface areas, and the result is converted into a percentage against the maximum value of the specific capacitance (MSC). The accessibility was obtained by the following equation:

$$
\text { Accessibility }=(\mathrm{SC} / \mathrm{S}) / \mathrm{MSC} \times 100,
$$

where the accessibility is a relative dimensionless variable. In this figure, the accessibility of many MP-series samples are shown altogether. The AC-series have from under $50 \%$ to $70 \%$ relative accessibility, assuming that the sample has the maximum capacitance. Therefore, this work confirms that MP-series samples have excellent capacitance properties, such as high conductivity, high specific capacitance, and high accessibility, etc., in spite of their small surface area.

\section{CONCLUSION}

Two kinds of samples, the powder-type AC-series and the fiber-type MP-series, were applied as polarizable electrodes for EDLCs. The sample electrodes made from the MP-series show much higher specific capacitance than those of the AC-series, although the MP-series materials have a smaller surface area. Furthermore, the MPseries materials show a higher capacitance of $46 \mathrm{~F} / \mathrm{g}$ at $10 \mathrm{~mA} / \mathrm{cm}^{2}$ than $40.9 \mathrm{~F} / \mathrm{g}$ at $1 \mathrm{~mA} / \mathrm{cm}^{2}$ discharge current density for the AC-series. Such a novel behavior of MPseries samples was investigated by various methods such as XRD, SEM, BET analysis, and pore size distribution by the image analysis of TEM photographs. Consequently, when the accessibility of the AC-series with that the MP-series is compared, the AC-series are found to have a relative low accessibility due to the morphological difference of the pores. That is, around $70 \%$ of the accessibility is found for the MP-series at their maximum capacitance. Besides, in the case of AC-a, it was shown that the accessibility is under half of that for the MP-series.

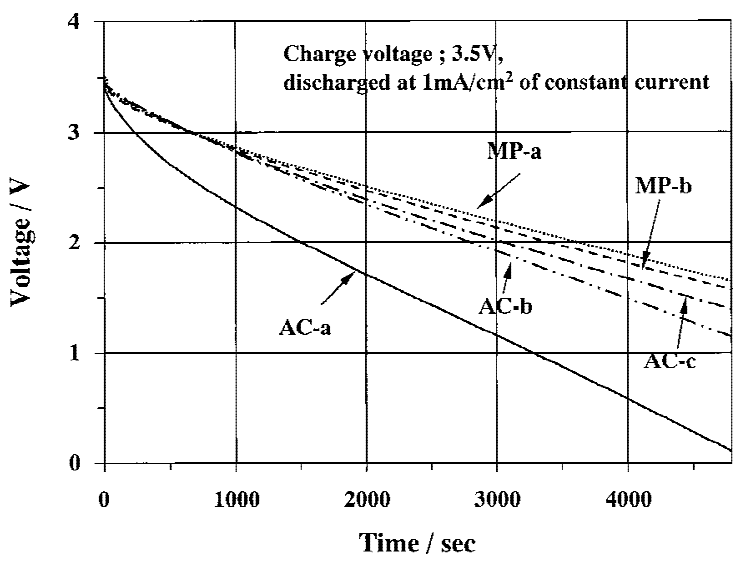

FIG. 7. Charge-discharge profiles of the AC-series and MP-series. Charging voltage is $3.5 \mathrm{~V}$, and discharging current density is $1 \mathrm{~mA} / \mathrm{cm}^{2}$. 


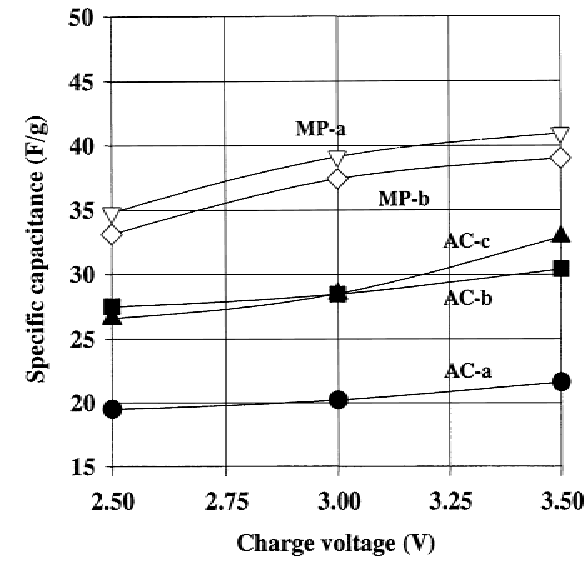

(a) (a-1) Current density, $1 \mathrm{~mA} / \mathrm{cm}^{2}$

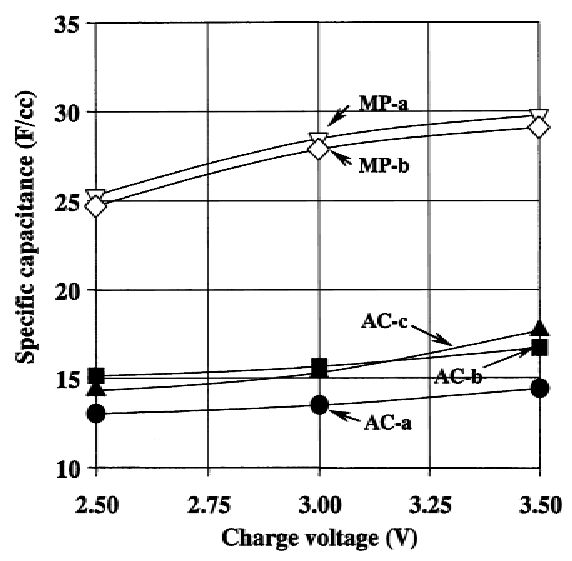

(b)

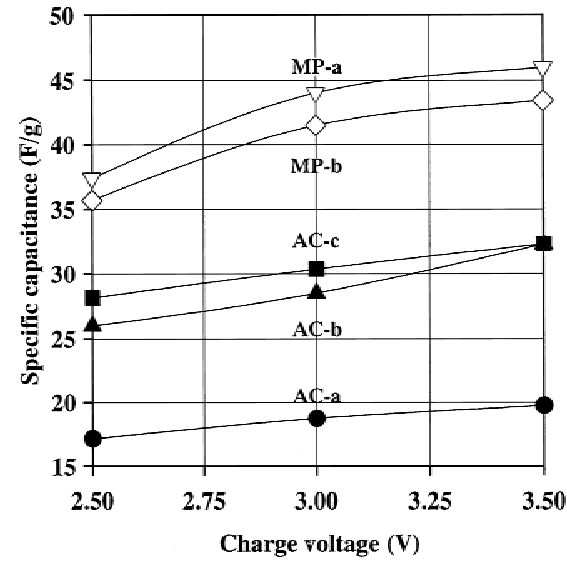

(a-2) Current density, $10 \mathrm{~mA} / \mathrm{cm}^{2}$

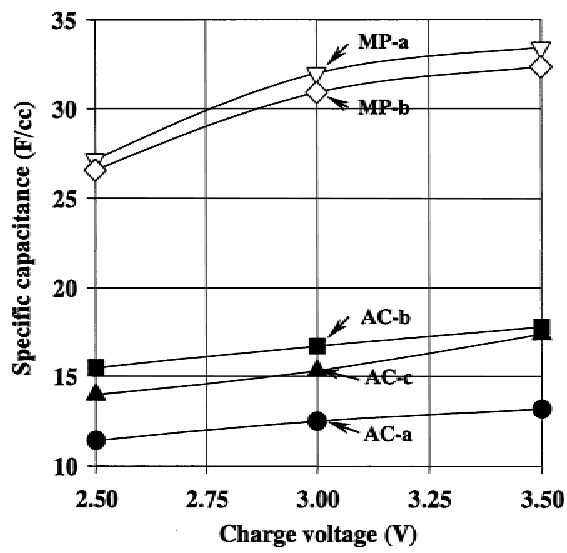

(b-2) Current density, $10 \mathrm{~mA} / \mathrm{cm}^{2}$

FIG. 8. Specific capacitance per (a) weight and (b) volume obtained at a constant current density of (1) $1 \mathrm{~mA} / \mathrm{cm}^{2}$ and (2) $10 \mathrm{~mA} / \mathrm{cm}^{2}$.

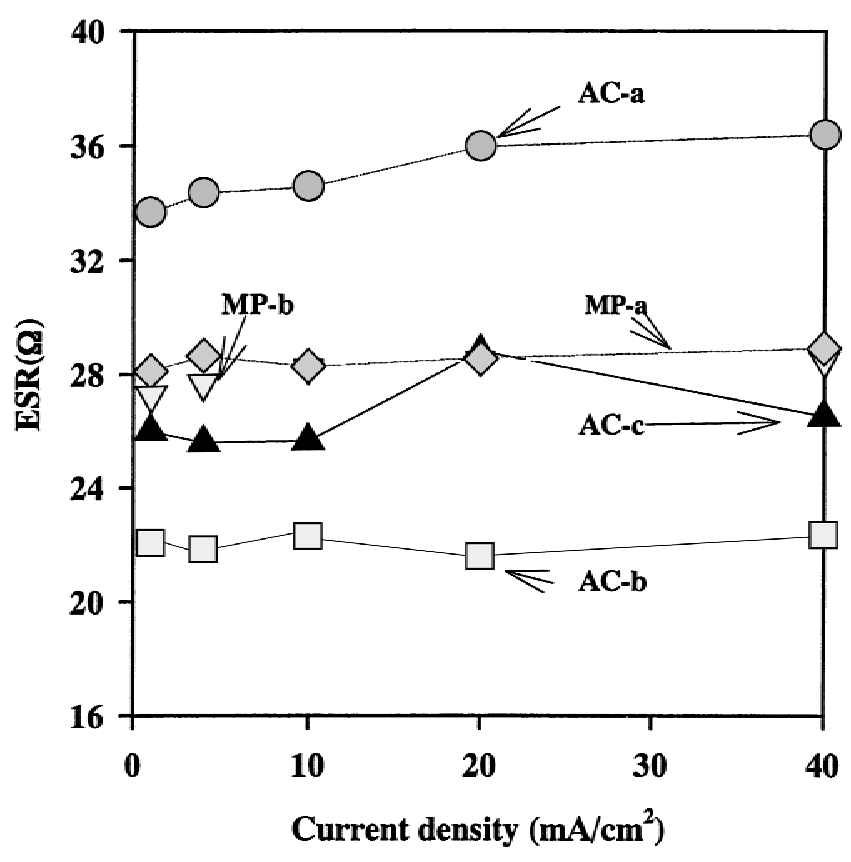

FIG. 9. The variation of equivalent series resistance (ESR) with current density for all the samples.

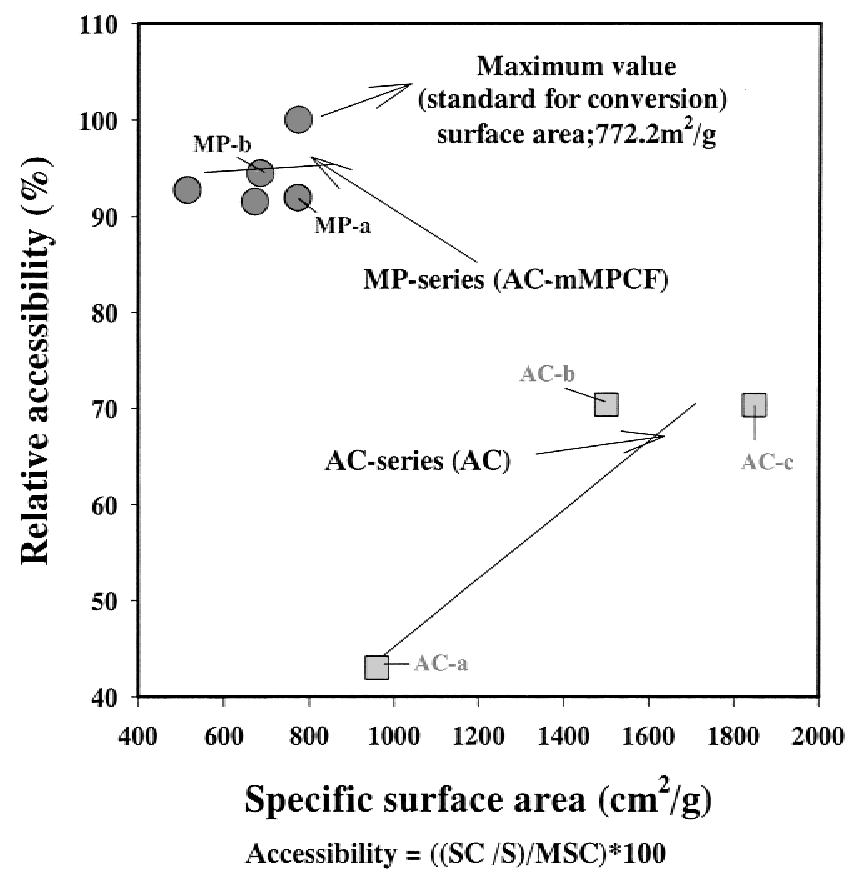

FIG. 10. Relative accessibility per surface area on the basis of the maximum value of the specific capacitance. 


\section{REFERENCES}

1. A. Nishino, Carbon 132, 57 (1988).

2. B.E. Conway, J. Electrochem. Soc. 138, 1539 (1991).

3. H.L.F. Von Helmholtz, Ann. Physik 89, 211 (1853).

4. H.L.F. Von Helmholtz, Ann. Physik 7, 337 (1879).

5. G. Gouy, J. Phys. Radium 9, 457 (1910).

6. D.L. Champman, Philos. Mag. 25, 475 (1913).

7. O. Stern, Z. Elektrochem. 30, 508 (1924).

8. B.E. Conway, Theory and Principles of Electrode Processes (Ronald, New York, 1965), Chaps. 4 and 5.

9. R. Payne, in Techniques of Electrochemistry, Vol. 1, edited by E. Yeager and A.J. Salkind (Wiley-Interscience, New York, 1972), p 43ff.

10. A. Nishino, A. Yoshida, and I. Tanahashi, U.S. Patent No. 4562 511 (1985).

11. S. Sekido, Y. Yoshino, T. Muranaka, and M. Mori, Denki Kagaku 48, 40 (1980).

12. R.C. Bansel, J-B. Donnet, and F. Stoeckli, Active Carbon (Dekker, New York, 1988).

13. L.B. Adams, E.A. Boucher, and D.H. Everett, Carbon 8, 76 (1970).
14. J.N. Bohra and K.S.W. Sing, Adsorption Sci. Tech. 2, 89 (1985).

15. G.G. Jayson and T.A. Lawless, J. Colloid Interface Sci. 86, 397 (1982).

16. K. Kinoshita, Carbon: Electrochemical and Physiochemical Properties (Wiley, New York, 1988), pp. 294-295.

17. M. Endo, K. Takeuchi, Y. Sasuda, K. Matsubayashi, K. Oshida, and M.S. Dresselhaus, Electronics and Communications in Japan, 77, 98-106 (1994).

18. K. Oshida, K. Kogiso, K. Matsubayashi, K. Takeuchi, S. Kobayashi, M. Endo, M.S. Dresselhaus, and G. Dresselhaus, J. Mater. Res. 10, 2507 (1995).

19. M. Endo, C. Kim, T. Karaki, T. Kasai, M.J. Matthews, S.D.M. Brown, M.S. Dresselhaus, T. Tamaki, and Y. Nishimura, Carbon 36, 1633 (1988).

20. Z. Weishauptova and J. Medek, Fuel 70, 235 (1991).

21. Z. Weishauptova, J. Medek, and Z. Vaverkova, Carbon, 32, 311 (1994).

22. R.S. Mikhali, S. Brunauer, and E.E. Boder, J. Colloid Interface Sci. 26, 45 (1968) 